\title{
ON THE AVERAGE SUM OF THE K-TH DIVISOR FUNCTION OVER VALUES OF QUADRATIC POLYNOMIALS
}

\author{
KOSTADINKA LAPKOVA and NIAN HONG ZHOU
}

\begin{abstract}
Let $F(\mathbf{x}) \in \mathbb{Z}\left[x_{1}, x_{2}, \ldots, x_{n}\right]$ be a quadratic polynomial in $n \geq 3$ variables with a nonsingular quadratic part. Using the circle method we derive an asymptotic formula for the sum

$$
\Sigma_{k, F}(X ; \mathcal{B})=\sum_{\mathbf{x} \in X \mathcal{B} \cap \mathbb{Z}^{n}} \tau_{k}(F(\mathbf{x})),
$$

for $X$ tending to infinity, where $\mathcal{B} \subset \mathbb{R}^{n}$ is an $n$-dimensional box such that $\min _{\mathbf{x} \in X \mathcal{B}} F(\mathbf{x}) \geq 0$ for all sufficiently large $X$, and $\tau_{k}(\cdot)$ is the $k$-th divisor function for any integer $k \geq 2$.
\end{abstract}

\section{Contents}

1 Introduction 1

2 The proof of Theorem $1.1 \quad 4$

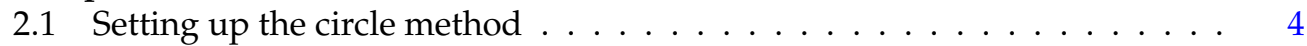

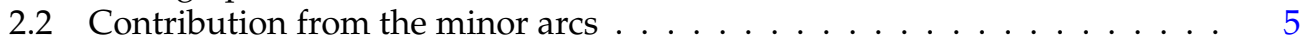

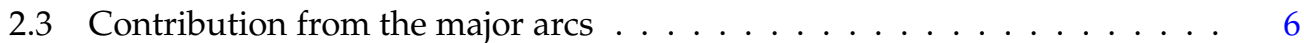

3 The estimates involving the $k$-th divisor function 9

4 The singular integral and series $\quad 14$

4.1 The singular integral . . . . . . . . . . . . . . . . . . 14

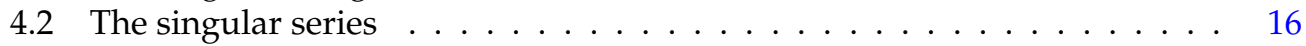

5 Final remarks $\quad 19$

\section{IntRoduction}

The $k$-th divisor function is a generalisation of the divisor function $\tau(m)=\sum_{d \mid m} 1$ which counts the number of ways $m$ can be written as a product of $k$ positive integer numbers. It is defined as

$$
\tau_{k}(m)=\#\left\{\left(x_{1}, x_{2}, \ldots, x_{k}\right) \in \mathbb{Z}_{+}^{k}: m=x_{1} x_{2} \ldots x_{k}\right\},
$$

where we assume that $\tau_{k}(0)=0$. For polynomials $F(\mathbf{x}) \in \mathbb{Z}\left[x_{1}, \ldots, x_{n}\right]$ consider the sums

$$
T_{k}(F(\mathbf{x}), X)=\sum_{|F(\mathbf{x})| \leq X} \tau_{k}(|F(\mathbf{x})|) .
$$

Understanding the average order of $\tau_{k}(m)$, as it ranges over sparse sequences of values taken by polynomials, i.e. of $T_{k}(F, X)$, is a problem that has received a lot of attention. 
The most studied case is naturally $k=2$. For $F(\mathbf{x})=F\left(x_{1}, x_{2}\right)$ a binary irreducible cubic form Greaves [8] showed that there exist real constants $c_{1}>0$ and $c_{2}$ depending only on $F$, such that

$$
T_{2}(F(\mathbf{x}), X)=c_{1} X^{2 / 3} \log X+c_{2} X^{2 / 3}+O_{\varepsilon, F}\left(X^{9 / 14+\varepsilon}\right),
$$

holds for any $\varepsilon>0$ as $X \rightarrow \infty$. If $F\left(x_{1}, x_{2}\right)$ is an irreducible quartic form, Daniel [6] proved that

$$
T_{2}(F(\mathbf{x}), X)=c_{1} X^{1 / 2} \log X+O_{F}\left(X^{1 / 2} \log \log X\right),
$$

where $c_{1}>0$ is a constant depending only on $F$. It seems that $\operatorname{deg} F=4$ is the limit of the current available methods treating divisor sums over binary forms. More related works on the cases $k=2$ and $n=2$ are e.g. la Bretèche and Browning [3], Browning [4] and $\mathrm{Yu}$ [17]. On the other hand, with their paper from 2012 Guo and Zhai [9] revived the interest toward estimating asymptotically $T_{2}(F(\mathbf{x}), X)$ for forms in $n \geq 3$ variables using the classical circle method. After many other papers extending [9] and dealing with diagonal forms, in a recent work Liu [11] obtained an asymptotic formula for $T_{2}(F(\mathbf{x}), X)$ for any nonsingular quadratic form $F$ in $n \geq 3$ variables.

For the cases when $k \geq 3$ there are only few results in the literature. Friedlander and Iwaniec [7] showed that

$$
\sum_{\substack{n_{1}^{2}+n_{2}^{6} \leq X \\ \operatorname{gcd}\left(n_{1}, n_{2}\right)=1}} \tau_{3}\left(n_{1}^{2}+n_{2}^{6}\right)=c X^{2 / 3}(\log X)^{2}+O\left(X^{2 / 3}(\log X)^{7 / 4}(\log \log X)^{1 / 2}\right),
$$

where $c$ is a constant. Daniel $[5,(4.5)]$ described an asymptotic formula for $T_{k}(F(\mathbf{x}), X)$ as $X \rightarrow \infty$ for any $k \geq 2$ for irreducible binary definite quadratic forms $F$ and in [5, (4.7)] he proved an asymptotic formula for $T_{3}(F(\mathbf{x}), X)$ as $X \rightarrow \infty$ for irreducible binary cubic forms $F$. Sun and Zhang [16], with the help of the circle method, obtained

$$
\sum_{1 \leq m_{1}, m_{2}, m_{3} \leq X} \tau_{3}\left(m_{1}^{2}+m_{2}^{2}+m_{3}^{2}\right)=c_{1} X^{3} \log ^{2} X+c_{2} X^{3} \log X+c_{3} X^{3}+O_{\varepsilon}\left(X^{11 / 4+\varepsilon}\right),
$$

where $c_{1}, c_{2}, c_{3}$ are constants and $\varepsilon$ is any positive number. Finally Blomer [2] proved an asymptotic formula for the sum $\Sigma_{k, F}(X ; \mathcal{B})$ defined in (1.2), for any $k \geq 2$, where $F(\mathbf{x})$ is a form of degree $k$ in $n=k-1$ variables, coming from incomplete norm form.

In this paper we investigate the average sum of the $k$-th divisor function over values of quadratic polynomials $F(\mathbf{x})$, not necessarily homogenous, in $n \geq 3$ variables for any $k \geq 2$. Every $n$-variables quadratic polynomial can be written as

$$
F(\mathbf{x})=\mathbf{x}^{T} Q \mathbf{x}+\mathbf{L}^{T} \mathbf{x}+N
$$

where $Q \in \mathbb{Z}^{n \times n}$ is a symmetric matrix, $\mathbf{L} \in \mathbb{Z}^{n}$ and $N \in \mathbb{Z}$. Our only additional requirement is that $Q$ is nonsingular. Let $\mathcal{B} \subset \mathbb{R}^{n}$ be an $n$-dimensional box (i.e. a certain product of intervals) such that $\min _{\mathbf{x} \in X \mathcal{B}} F(\mathbf{x}) \geq 0$ for all sufficiently large $X$, and for each integer $k \geq 2$, consider the sum

$$
\Sigma_{k, F}(X ; \mathcal{B})=\sum_{\mathbf{x} \in X \mathcal{B} \cap \mathbb{Z}^{n}} \tau_{k}(F(\mathbf{x})),
$$

as $X$ tends to infinity. Let us also use the following notation for $q \in \mathbb{Z}_{+}$

$$
\varrho_{F}(q)=\frac{1}{q^{n-1}} \#\{\mathbf{h} \quad(\bmod q): F(\mathbf{h}) \equiv 0 \bmod q\} .
$$


Our main result is the following.

Theorem 1.1. Let $F(\mathbf{x})$ and $\Sigma_{k, F}(X ; \mathcal{B})$ be defined as in (1.1) and (1.2), respectively, where $Q$ is a nonsingular matrix. Then for any $\varepsilon>0$ there exist real constants $C_{k, 0}(F), C_{k, 1}(F), \ldots$, and $C_{k, k-1}(F)$, such that for $X$ tending to infinity we have the asymptotic formula

$$
\Sigma_{k, F}(X ; \mathcal{B})=\sum_{r=0}^{k-1} C_{k, r}(F) \int_{X \mathcal{B}}(\log F(\mathbf{t}))^{r} d \mathbf{t}+O\left(X^{n-\frac{n-2}{n+2} \min \left(1, \frac{4}{k+1}\right)+\varepsilon}\right),
$$

where the implied constant depends on $F, k, \mathcal{B}$ and $\varepsilon$, and

$$
C_{k, r}(F)=\frac{1}{r !} \sum_{t=0}^{k-r-1} \frac{1}{t !}\left(\left.\frac{d^{t} L(s ; k, F)}{d s^{t}}\right|_{s=1}\right) \operatorname{Res}_{s=1}\left((s-1)^{r+t} \zeta(s)^{k}\right) .
$$

The function $L(s ; k, F)$ has the Euler product presentation

$$
L(s ; k, F)=\prod_{p}\left(\sum_{\ell \geq 0} \frac{\varrho_{F}\left(p^{\ell}\right)\left(\tau_{k}\left(p^{\ell}\right)-p^{s-1} \tau_{k}\left(p^{\ell-1}\right)\right)}{p^{\ell s}}\right)\left(\frac{\left(1-p^{-s}\right)^{k}}{1-p^{-1}}\right),
$$

with $\tau_{k}(x):=0$ for all $x \notin \mathbb{Z}$, and it is absolutely convergent for all $\mathfrak{R}(s)>1 / 2$. In particular, the main term has a positive leading coefficient:

$$
C_{k, k-1}(F)=\frac{1}{(k-1) !} \prod_{p}\left(\sum_{\ell \geq 0} \frac{\varrho_{F}\left(p^{\ell}\right) \tau_{k-1}\left(p^{\ell}\right)}{p^{\ell}}\right)\left(1-\frac{1}{p}\right)^{k-1}>0 .
$$

First of all, we remark that since $F(\mathbf{x})$ has a nonsingular quadratic part, the set of all zeros of $F(\mathbf{x})=0$ has a Lebesgue measure 0 , so that the logarithm function in the integrals in the terms is well defined. Note that we provide a formula with $k$ terms, where one can easily see that the main term is of magnitude $X^{n}(\log X)^{k-1}$ (when $r=k-1$ ) and the last secondary term is of magnitude $X^{n}$ (when $r=0$ ). Thus the error term is indeed of a smaller rate.

Using Theorem 1.1, one can get the asymptotic formula for $\Sigma_{2, F}(X, \mathcal{B})$ in the most studied case of $k=2$. This recreates and extends the main Theorem of Liu [11] also for non-homogenous quadratic polynomials, but also provides different expressions for the coefficients. Naturally, they can be also computed explicitly for specific polynomials, a goal we have not pursued in the current paper. Theorem 1.1 also extends the formula [5, (4.5)] of Daniel to quadratic polynomials in more than 2 variables, further, it elucidates the form of the involved coefficients.

Notations. The symbols $\mathbb{Z}_{+}, \mathbb{Z}$ and $\mathbb{R}$ denote the positive integers, the integers and the real numbers, respectively. $e(z):=e^{2 \pi i z}, \zeta(s)=\sum_{n \geq 1} n^{-s}$ is the Riemann zeta function, the letter $p$ always denotes a prime. We make use of the $\varepsilon$-convention: whenever $\varepsilon$ appears in a statement, it is asserted that the statement is true for all real $\varepsilon$. This allows us to write $x^{\varepsilon} \log x \ll x^{\varepsilon}$ and $x^{2 \varepsilon} \ll x^{\varepsilon}$, for example. Furthermore, if not specially specified, all the implied constants of this paper in $O$ and $\ll$ depend on $F, k, \mathcal{B}$ and $\varepsilon$. 


\section{The PROOF OF THEOREM 1.1}

\subsection{SETTING UP THE CIRCLE METHOD}

The primary technique used in the proof of the main theorem is the circle method and more precisely its treatment by Pleasants [13]. The recent work on quadratic forms in $n \geq 3$ variables of Liu [11] uses the same circle method techniques, i.e. Weyl differencing, that were already used for general quadratic multivariable polynomials by Pleasants.

For the real $X$ from the definition (1.2) let $L \ll X$ be a positive real parameter which we will choose later in a suitable way, let $a, q \in \mathbb{Z}, 0 \leq a<q \leq L$ and $\operatorname{gcd}(a, q)=1$. Then we define the intervals

$$
\mathfrak{M}_{a, q}(L):=\left\{\alpha \in[0,1]:\left|\alpha-\frac{a}{q}\right| \leq \frac{L}{q X^{2}}\right\} .
$$

The set of the major arcs is then the union

$$
\mathfrak{M}(L)=\bigsqcup_{\substack{0 \leq a<q \leq L \\ \operatorname{gcd}(a, q)=1}} \mathfrak{M}_{a, q}(L),
$$

and the set of the minor arcs is the complement $\mathfrak{m}(L)=[0,1] \backslash \mathfrak{M}(L)$.

We further define the following exponential sums for $\alpha \in \mathbb{R}$

$$
S(\alpha)=\sum_{\mathbf{x} \in X \mathcal{B} \cap \mathbb{Z}^{n}} e(F(\mathbf{x}) \alpha)
$$

and

$$
T(\alpha, Y)=\sum_{0 \leq m \leq Y} \tau_{k}(m) e(m \alpha) .
$$

Then, by the well-known identity for $u \in \mathbb{Z}$

$$
\int_{0}^{1} e(u \alpha) d \alpha=\left\{\begin{array}{lll}
1 & \text { if } & u=0 \\
0 & \text { if } & u \neq 0
\end{array}\right.
$$

we have

$$
\begin{aligned}
\sum_{k, F}(X ; \mathcal{B}) & =\sum_{\mathbf{x} \in X \mathcal{B} \cap \mathbb{Z}^{n}} \tau_{k}(F(\mathbf{x})) \\
& =\int_{0}^{1} S(\alpha) T\left(-\alpha, C_{F, \mathcal{B}}(X)\right) d \alpha \\
& =\int_{\mathfrak{M}(L)} S(\alpha) T\left(-\alpha, C_{F, \mathcal{B}}(X)\right) d \alpha+\int_{\mathfrak{m}(L)} S(\alpha) T\left(-\alpha, C_{F, \mathcal{B}}(X)\right) d \alpha \\
& =I_{\mathfrak{M}(L)}+I_{\mathfrak{m}(L),}
\end{aligned}
$$

where

$$
C_{F, \mathcal{B}}(X):=\max _{\mathbf{x} \in X \mathcal{B}}|F(\mathbf{x})|=X^{2} \max _{\mathbf{x} \in \mathcal{B}}\left|\mathbf{x}^{T} Q \mathbf{x}\right|+O(X) \asymp X^{2} .
$$

We shall prove in Section 2.2 that for the contribution from the minor arcs we have

$$
I_{\mathfrak{m}(L)} \ll X^{n+\varepsilon} L^{1-n / 2},
$$


as long as $L \ll X$. Already here we see that we need to require that the number of variables satisfy $n \geq 3$ in order to have an error term of a smaller magnitude than $O\left(X^{n}\right)$. Further, in Section 2.3 we will show that

$$
I_{\mathfrak{M}(L)}-\sum_{r=0}^{k-1} C_{k, r}(F) \int_{X \mathcal{B}}(\log (F(\mathbf{t})))^{r} d \mathbf{t} \ll X^{n+\varepsilon}\left(L^{1-n / 2}+L^{2} X^{-\min \left(1, \frac{4}{k+1}\right)}\right) .
$$

Here for $r=0,1, \ldots, k-1$,

$$
C_{k, r}(F)=\sum_{q=1}^{\infty} \beta_{k, r}(q) S_{F}(q)
$$

where

$$
\begin{gathered}
S_{F}(q)=\sum_{\substack{a \in[1, q] \cap \mathbb{Z} \\
\operatorname{gcd}(a, q)=1}} q^{-n} \sum_{\mathbf{h} \in[1, q]^{n} \cap \mathbb{Z}^{n}} e\left(\frac{a}{q} F(\mathbf{h})\right), \\
\beta_{k, r}(q)=\frac{1}{r !} \sum_{t=0}^{k-r-1} \frac{1}{t !} \operatorname{Res}\left((s-1)^{r+t} \zeta(s)^{k}\right)\left(\left.\frac{d^{t} \Phi_{k}(q, s)}{d s^{t}}\right|_{s=1}\right),
\end{gathered}
$$

and the analytic function $\Phi_{k}(q, s)$ is defined by Lemma 3.2. We further consider the function

$$
L(s ; k, F)=\sum_{q \geq 1} \Phi_{k}(q, s) S_{F}(q)
$$

In Subsection 4.2 we prove that it satisfies

$$
L(s ; k, F)=\prod_{p}\left(\sum_{\ell \geq 0} \frac{\varrho_{F}\left(p^{\ell}\right)\left(\tau_{k}\left(p^{\ell}\right)-p^{s-1} \tau_{k}\left(p^{\ell-1}\right)\right)}{p^{\ell s}}\right)\left(\frac{\left(1-p^{-s}\right)^{k}}{1-p^{-1}}\right),
$$

with $\tau_{k}(x):=0$ for all $x \notin \mathbb{Z}$.

Then Theorem 1.1 follows from (2.2), (2.3) and (2.7), after choosing $L=X^{\frac{2}{n+2}} \min \left(1, \frac{4}{k+1}\right)$.

\subsection{CONTRIBUTION FROM THE MINOR ARCS}

Clearly, if the positive real numbers $L$ and $L^{\prime}$ satisfy $L \leq L^{\prime}$, then $\mathfrak{M}(L) \subset \mathfrak{M}\left(L^{\prime}\right)$, and if $L \geq X$, then $[0,1] \subset \mathfrak{M}(L)$ follows from Dirichlet's approximation theorem.

We further define

$$
\mathscr{F}(L)=\mathfrak{M}(2 L) \backslash \mathfrak{M}(L) .
$$

Then for a given positive number $L<X / 2$,

$$
[0,1] \subset \mathfrak{M}(L) \sqcup \bigsqcup_{0 \leq j<N} \mathscr{F}\left(2^{j} L\right),
$$

where $N$ is the smallest integer greater than or equal to $(\log (X / L)) / \log 2$. Clearly, the set of the small arcs then satisfy

$$
\mathfrak{m}(L) \subset \bigsqcup_{0 \leq j<N} \mathscr{F}\left(2^{j} L\right) .
$$

To prove the estimate (2.2) over the minor arcs, we would use separate estimates of the two components $S(\alpha)$ and $T(\alpha, X)$ when $\alpha \in \mathscr{F}(L)$. We first state the following result. 
Lemma 2.1. For all positive numbers $L \ll X$,

$$
\sup _{\alpha \in \mathscr{F}(L)}|S(\alpha)| \ll X^{n+\varepsilon} L^{-n / 2} .
$$

Proof. This estimate was done by Pleasants [13] even for the range $L \ll X(\log X)^{1 / 4}$. In the first equation of p.138 [13] he proves that for $\alpha \in \mathscr{F}(L)$ we have

$$
|S(\alpha)| \leq X^{n}(\log X)^{n} L^{-r / 2},
$$

where $r \geq 3$ is the rank of $Q$, and in our case we have assumed that $r=n$.

We also need the following estimate.

Lemma 2.2. For all positive numbers $L \ll X$,

$$
\int_{\mathscr{F}(L)}\left|T\left(-\alpha, C_{F, \mathcal{B}}(X)\right)\right| d \alpha \ll X^{\varepsilon} L
$$

Proof. By Cauchy's inequality, and using the definition of the major arcs (2.1), we have

$$
\begin{aligned}
\int_{\mathscr{F}(L)}\left|T\left(-\alpha, C_{F, \mathcal{B}}(X)\right)\right| d \alpha & \ll|\mathscr{F}(L)|^{1 / 2}\left(\int_{0}^{1}\left|T\left(-\alpha, C_{F, \mathcal{B}}(X)\right)\right|^{2} d \alpha\right)^{1 / 2} \\
& \ll|\mathfrak{M}(2 L)|^{1 / 2}\left(\left(\sum_{1 \leq n \leq C_{F, \mathcal{B}}(X)} \tau_{k}(n)\right)^{2}\right)^{1 / 2} \\
& \ll\left(\sum_{1 \leq q \leq L} \frac{2 L}{q X^{2}} \varphi(q)\right)^{1 / 2} X^{1+\varepsilon} \ll X^{-1+1+\varepsilon} L \ll X^{\varepsilon} L,
\end{aligned}
$$

where we also applied the well known bound $\tau_{k}(n) \ll_{k} n^{\varepsilon}$ and the trivial $\varphi(q) / q \leq 1$.

Now the estimate (2.2) over the minor arcs follow from (2.8), Lemma 2.1 and Lemma 2.2 , namely

$$
\begin{aligned}
I_{\mathfrak{m}(L)} & \ll \sum_{0 \leq j<N} \int_{\mathscr{F}(2 j L)}\left|S(\alpha) T\left(-\alpha, C_{F, \mathcal{B}}(X)\right)\right| d \alpha \\
& \ll \sum_{0 \leq j<N} \sup _{\alpha \in \mathscr{F}\left(2^{j} L\right)}|S(\alpha)| \int_{\mathscr{F}\left(2^{j} L\right)}\left|T\left(-\alpha, C_{F, \mathcal{B}}(X)\right)\right| d \alpha \\
& \ll \sum_{0 \leq j<N} X^{n+\varepsilon}\left(2^{j} L\right)^{-n / 2}\left(X^{\varepsilon} 2^{j} L\right) \ll X^{n+\varepsilon} L^{1-n / 2},
\end{aligned}
$$

where we used that $N \ll \log X$.

\subsection{CONTRIBUTION FROM THE MAJOR ARCS}

In this subsection we have $\alpha \in \mathfrak{M}_{a, q}(L)$, and we shall write $\beta=\alpha-a / q$ for the coprime integers $a$ and $q,|\beta| \leq L /\left(q X^{2}\right)$ and $1 \leq q \leq L$. In order to prove the asymptotic formula (2.3), we need the following statements. 
Lemma 2.3. For $\alpha \in \mathfrak{M}_{a, q}(L)$, and $\beta=\alpha-a / q$, we have

$$
S(\alpha)=q^{-n} S_{F}(q, a) \int_{X \mathcal{B}} e(F(\mathbf{t}) \beta) d \mathbf{t}+O_{\mathcal{B}, F}\left(L X^{n-1}\right),
$$

where

$$
S_{F}(q, a)=\sum_{\mathbf{h} \in[1, q]^{n} \cap \mathbb{Z}^{n}} e\left(\frac{a}{q} F(\mathbf{h})\right) .
$$

Proof. To prove this result we only need to adjust the last equation in the proof of [13, Lemma 8] with the upper bounds $\beta \leq L /\left(q X^{2}\right)$ and $q \leq L$. Note that Pleasant does the analysis over a quadratic polynomial with linear coefficients which can depend on $X$. We are dealing with a quadratic $F$ with fixed coefficients, which makes the proof even easier.

Lemma 2.4. Let $S_{F}(q, a)$ be defined as in Lemma 2.3. We have

$$
S_{F}(q, a) \ll_{F} q^{n / 2+\varepsilon},
$$

where the implied constant is independent of $a$ and $q$

Proof. This is [13, Lemma 10].

We further need the following two statements. The first one gives a general asymptotic representation of $T(\alpha, \gamma)$ and the second one estimates the part of the singular integral coming from the major arcs. The proofs of Lemma 2.5 and Lemma 2.6 will be given in Section 3 and Section 4.1, respectively.

Lemma 2.5. Let $Y \asymp X^{2}$. We have

$$
T(\alpha, Y)=\sum_{r=0}^{k-1} \beta_{k, r}(q) \int_{0}^{Y}(\log u)^{r} e(u \beta) d u+O_{k, \varepsilon}\left(L X^{2-\frac{4}{k+1}+\varepsilon}\right) .
$$

where for $r=0,1, \ldots, k-1$,

$$
\beta_{k, r}(q)=\frac{1}{r !} \sum_{t=0}^{k-r-1} \frac{1}{t !} \operatorname{Res}_{s=1}\left((s-1)^{r+t} \zeta(s)^{k}\right)\left(\left.\frac{d^{t} \Phi_{k}(q, s)}{d s^{t}}\right|_{s=1}\right) \ll q^{-1+\varepsilon} .
$$

with $\Phi_{k}(q, s)$ defined by Lemma 3.2. In particular,

$$
T(\alpha, Y) \ll q^{-1+\varepsilon} X^{2+\varepsilon} .
$$

Lemma 2.6. We have

$$
\int_{|\beta| \leq L / q X^{2}} d \beta \int_{X \mathcal{B}} d \mathbf{t} \int_{0}^{C_{F \in \mathcal{B}}(X)} e((F(\mathbf{t})-u) \beta)(\log u)^{r} d u=\int_{X \mathcal{B}}(\log F(\mathbf{t}))^{r} d \mathbf{t}+O\left(\frac{q^{n / 2} X^{n+\varepsilon}}{L^{n / 2}}\right) .
$$


DiVISOR FUNCTION OVER VALUES OF QUADRATIC POLYNOMIALS

We now prove the asymptotic formula (2.3). Using (2.1) we get

$$
\begin{aligned}
I_{\mathfrak{M}(L)} & =\int_{\mathfrak{M}(L)} S(\alpha) T\left(-\alpha, C_{F, \mathcal{B}}(X)\right) d \alpha \\
& =\sum_{q \leq L} \sum_{\substack{0 \leq a<q \\
\operatorname{gcd}(a, q)=1}} \int_{|\beta| \leq L / q X^{2}} S(\alpha) T\left(-\alpha, C_{F, \mathcal{B}}(X)\right) d \alpha \\
& =: \sum_{q \leq L} \sum_{\substack{0 \leq a<q \\
\operatorname{gcd}(a, q)=1}} I_{q, a} .
\end{aligned}
$$

Since $1 \leq q \leq L \ll X$, we have

$$
\begin{aligned}
\mathcal{I}_{q, a}= & \int_{|\beta| \leq L / q X^{2}} S(\alpha) T\left(-\alpha, C_{F, \mathcal{B}}(X)\right) d \beta \\
= & \int_{|\beta| \leq L / q X^{2}}\left(\frac{S_{F}(q, a)}{q^{n}} \int_{X \mathcal{B}} e(F(\mathbf{t}) \beta) d \mathbf{t}\right) T\left(-\alpha, C_{F, \mathcal{B}}(X)\right) d \beta \\
& +O\left(\int_{|\beta| \leq L / q X^{2}}\left(L X^{n-1}\right) q^{-1+\varepsilon} X^{2+\varepsilon} d \beta\right),
\end{aligned}
$$

by Lemma 2.3. Further, by applying Lemma 2.4, Lemma 2.5 and Lemma 2.6, we get

$$
\begin{aligned}
\mathcal{I}_{q, a}= & \sum_{r=0}^{k-1} \frac{S_{F}(q, a) \beta_{k, r}(q)}{q^{n}} \int_{|\beta| \leq L / q X^{2}} d \beta \int_{X \mathcal{B}} e(F(\mathbf{t}) \beta) d \mathbf{t} \int_{0}^{C_{F, \mathcal{B}}(X)}(\log u)^{r} e(-u \beta) d u \\
& +O\left(\int_{|\beta| \leq L / q X^{2}} q^{-n / 2+\varepsilon} X^{n}\left(L X^{2-\frac{4}{k+1}+\varepsilon}\right) d \beta+\frac{L^{2} X^{n-1+\varepsilon}}{q^{2}}\right) \\
= & \sum_{r=0}^{k-1} \frac{S_{F}(q, a) \beta_{k, r}(q)}{q^{n}} \int_{X \mathcal{B}}(\log F(\mathbf{t}))^{r} d \mathbf{t}+O\left(\frac{X^{n+\varepsilon}}{q L^{n / 2}}+\frac{X^{n-\frac{4}{k+1}+\varepsilon} L^{2}}{q^{1+n / 2}}+\frac{L^{2} X^{n-1+\varepsilon}}{q^{2}}\right) .
\end{aligned}
$$

Recall the notation (2.4) and note that

$$
S_{F}(q)=\sum_{\substack{a \in[1, q] \cap \mathbb{Z} \\ \operatorname{gcd}(a, q)=1}} q^{-n} S_{F}(q, a) .
$$

Then after summing over all $1 \leq q \leq L$ and $1 \leq a<q, \operatorname{gcd}(a, q)=1$, the major arcs $\mathfrak{M}(L)$ contribute

$$
\begin{aligned}
I_{\mathfrak{M}(L)}= & \sum_{q \geq 1} S_{F}(q) \sum_{r=0}^{k-1} \beta_{k, r}(q) \int_{X \mathcal{B}}(\log F(\mathbf{t}))^{r} d \mathbf{t}+O\left(\sum_{q>L} q^{-1+\varepsilon}\left|S_{F}(q)\right| X^{n+\varepsilon}\right) \\
& +O\left(X^{n+\varepsilon} L^{1-n / 2}+X^{n-\frac{4}{k+1}+\varepsilon} L^{2}+L^{2} X^{n-1+\varepsilon}\right) \\
= & \sum_{r=0}^{k-1} C_{k, r}(F) \int_{X \mathcal{B}}(\log (F(\mathbf{t})))^{r} d \mathbf{t}+O\left(X^{n+\varepsilon} E\right),
\end{aligned}
$$


with

$$
E=L^{1-n / 2}+L^{2}\left(X^{-1}+X^{-\frac{4}{k+1}}\right) \ll L^{1-n / 2}+L^{2} X^{-\min \left(\frac{4}{k+1}, 1\right)} .
$$

Note that at this step, and at few other places, in order to control the error terms we necessarily have $n \geq 3$. This completes the proof of (2.3).

\section{THE ESTIMATES INVOLVING THE $k$-TH DIVISOR FUNCTION}

The usual technique in estimating asymptotically through the circle method average sums similar to $\Sigma_{k, F}(X, \mathcal{B})$, is the application of non-trivial average estimates of the specific arithmetic function over arithmetic progressions ( e.g. [9], [10], [11]). Thus in order to prove Lemma 2.5 we first need the following result.

Lemma 3.1. Let $h, q$ be integers such that $1 \leq h \leq q$ and $\operatorname{gcd}(h, q)=\delta$. Then for each real number $x>1, q \leq x^{\frac{2}{k+1}}$ and $\varepsilon>0$, we have

$$
A_{k}(x ; h, q):=\sum_{\substack{m \leq x \\ m \equiv h(\bmod q)}} \tau_{k}(m)=M_{k}(x ; h, q)+O_{k, \varepsilon}\left(x^{1-\frac{2}{k+1}+\varepsilon}\right),
$$

where

$$
M_{k}(x ; h, q)=\operatorname{Res}_{s=1}\left(\zeta(s)^{k} \frac{x^{s}}{s} f_{k}(q, \delta, s)\right)
$$

with

$$
f_{k}(q, \delta, s)=\frac{1}{\varphi(q / \delta) \delta^{s}}\left(\sum_{d \mid(q / \delta)} \frac{\mu(d)}{d^{s}}\right)^{k} \sum_{d_{1} d_{2} \ldots d_{k}=\delta} \sum_{\substack{t_{i} \mid\left(\prod_{j=i+1}^{k} d_{j}\right) \\ \operatorname{gc}\left(t_{i}, q / \delta\right)=1 \\ i=1,2, \ldots, k}} \frac{\mu\left(t_{1}\right) \ldots \mu\left(t_{k}\right)}{\left(t_{1} \ldots t_{k}\right)^{s}},
$$

where $d_{1}, d_{2}, \ldots, d_{k}$ are positive integers and the empty product $\prod_{j=k+1}^{k} d_{k}:=1$.

Proof. This lemma is essentially due to Smith [15], and we only adjust it for our purposes. We will extend easily [15, Theorem 3], which covers the case when $h$ and $q$ are coprime, to any $h$ and $q$. First, equation (30) of [15] states that

$$
A_{k}(x ; h, q)=\sum_{d_{1} d_{2} \ldots d_{k}=\delta} \sum_{\substack{t_{i}\left(\prod_{j=i+1}^{k} d_{j}\right) \\ i=1, \ldots, k \\ \operatorname{gcd}\left(t_{1} t_{2} \ldots \ldots, q, \delta\right)=1}} \mu(\mathbf{t}) A_{k}\left(\frac{x}{\delta t_{1} t_{2} \ldots t_{k}} ; \overline{t_{1} t_{2} \ldots t_{k}} \frac{h}{\delta}, \frac{q}{\delta}\right),
$$

where $d_{1}, d_{2}, \ldots, d_{r}$ are positive integers, $\mu(\mathbf{t})=\prod_{j=1}^{k} \mu\left(t_{j}\right)$ and $\bar{m}$ is the multiplicative inverse of $m$ modulo $q$. Then Theorem 3 of [15] states that

$$
A_{k}(x ; h, q)=M_{k}(x ; h, q)+\Delta_{k}(x ; h, q),
$$

where

$$
M_{k}(x ; h, q)=\sum_{d_{1} d_{2} \ldots d_{k}=\delta} \sum_{\substack{t_{i}\left(\prod_{i=i+1}^{k} d_{j}\right) \\ \operatorname{gcd}\left(t_{i}, q / \delta\right)=1 \\ i=1,2, \ldots, k}} \mu(\mathbf{t}) \frac{x}{\delta t_{1} t_{2} \ldots t_{k}} P_{k}\left(\log \left(\frac{x}{\delta t_{1} t_{2} \ldots t_{k}}\right), \frac{q}{\delta}\right)
$$


and

$$
\begin{aligned}
\Delta_{k}(x ; h, q)= & \sum_{d_{1} d_{2} \ldots d_{k}=\delta} \sum_{\substack{t_{i} \mid\left(\prod_{j=i+1}^{k} d_{j}\right) \\
\operatorname{gcd}\left(t_{i}, q / \delta\right)=1 \\
i=1,2, \ldots, k}} \mu(\mathbf{t})\left(D_{k}\left(0 ; \overline{t_{1} \ldots t_{k}} \frac{h}{\delta}, \frac{q}{\delta}\right)\right) \\
& +\sum_{d_{1} \ldots d_{k}=\delta} \sum_{\substack{t_{i}\left(\prod_{j=i+1}^{k} d_{j}\right) \\
\operatorname{gcd}\left(t_{i}, q / \delta\right)=1 \\
i=1,2, \ldots, k}} \mu(\mathbf{t})\left(O\left(\left(\frac{x}{\delta t_{1} \ldots t_{k}}\right)^{\frac{k-1}{k+1}} \tau_{k}\left(\frac{q}{\delta}\right) \log ^{k-1}(2 x)\right)\right) .
\end{aligned}
$$

Here $P_{k}(\log x, q)$ is a polynomial in $\log x$ of degree $k-1$ and $D_{k}(s ; h, q)$ is the Dirichlet series corresponding to the sum $A_{k}(x ; h, q)$. By the definition of $P_{k}(\log x, q)$, namely [15, (13)], and the analysis of $D_{k}(s ; h, q)$ given in particular in $[15,(21)]$, it is easily seen that

$$
x P_{k}(\log x, q)=\frac{1}{\varphi(q)} \operatorname{Res}_{s=1}\left(\left(\zeta(s) \sum_{d \mid q} d^{-s} \mu(d)\right)^{k} \frac{x^{s}}{s}\right) .
$$

Hence

$$
\begin{aligned}
M_{k}(x ; h, q) & =\sum_{d_{1} \ldots d_{k}=\delta} \sum_{\substack{t_{i} \mid\left(\prod_{j=i+1}^{k} d_{j}\right) \\
\operatorname{gcd}\left(t_{i}, q / \delta\right)=1 \\
i=1,2, \ldots, k}} \frac{\mu\left(t_{1}\right) \ldots \mu\left(t_{k}\right)}{\varphi(q / \delta)} \operatorname{Res}_{s=1}\left(\left(\zeta(s) \sum_{d \mid(q / \delta)} \frac{\mu(d)}{d^{s}}\right)^{k} \frac{x^{s} / s}{\left(\delta t_{1} \ldots t_{k}\right)^{s}}\right) \\
& =\operatorname{Res}_{s=1}\left(\frac{\zeta(s)^{k} x^{s} / s}{\varphi(q / \delta) \delta^{s}}\left(\sum_{d \mid(q / \delta)} \frac{\mu(d)}{d^{s}}\right)^{k} \sum_{d_{1} d_{2} \ldots d_{k}=\delta} \sum_{\substack{t_{i} \mid\left(\prod_{j=i+1}^{k} d_{j}\right) \\
\operatorname{gcd}\left(t_{i}, q / \delta\right)=1 \\
i=1,2, \ldots, k}} \frac{\mu\left(t_{1}\right) \ldots \mu\left(t_{k}\right)}{\left(t_{1} \ldots t_{k}\right)^{s}}\right) .
\end{aligned}
$$

Thus the main term is

$$
M_{k}(x ; h, q)=\operatorname{Res}_{s=1}\left(\zeta(s)^{k} \frac{x^{s}}{s} f_{k}(q, \delta, s)\right),
$$

where, as defined in the statement of the lemma, we have

$$
f_{k}(q, \delta, s)=\frac{1}{\varphi(q / \delta) \delta^{s}}\left(\sum_{d \mid(q / \delta)} \frac{\mu(d)}{d^{s}}\right)^{k} \sum_{d_{1} d_{2} \ldots d_{k}=\delta} \sum_{\substack{t_{i} \mid\left(\prod_{j=i+1}^{k} d_{j}\right) \\ \operatorname{gdd}\left(t_{i}, q / \delta\right)=1 \\ i=i_{2,2, \ldots, k}}} \frac{\mu\left(t_{1}\right) \ldots \mu\left(t_{k}\right)}{\left(t_{1} \ldots t_{k}\right)^{s}} .
$$

Smith [15] conjectured the validity of the estimate $D_{k}(0, h, q) \ll q^{\frac{k-1}{2}+\varepsilon}$ for any $(q, h)=1$. This was later affirmed by Matsumoto [12]. Therefore we have the bound

$$
\begin{aligned}
\Delta_{k}(x ; h, q) & \ll \sum_{d_{1} \ldots d_{k}=\delta} \sum_{t_{i} \mid\left(\prod_{j=i+1}^{k} d_{j}\right)}\left|\mu\left(t_{1}\right) \ldots \mu\left(t_{k}\right)\right|\left((q / \delta)^{\frac{k-1}{2}+\varepsilon}+q^{\varepsilon} x^{\frac{k-1}{k+1}+\varepsilon}\right) \\
& \ll{ }_{k}\left(q^{\frac{k-1}{2}+\varepsilon}+x^{\frac{k-1}{k+1}+\varepsilon}\right) \sum_{d_{1} \ldots d_{k}=\delta} \tau(\delta)^{k-1} \ll x^{1-\frac{2}{k+1}+\varepsilon},
\end{aligned}
$$

using $q \leq x^{\frac{2}{k+1}}$ and $\tau_{k}(\delta) \ll \delta^{\varepsilon}$. This completes the proof of the lemma. 
Lemma 3.2. Let $q \geq 1$ be an integer, $(a, q)=1$ and denote $\delta=(h, q)$. Also let $f_{k}(q, \delta, s)$ be defined as in Lemma 3.1. Define

$$
\Phi_{k, a}(q, s)=\sum_{h=1}^{q} e\left(-\frac{a h}{q}\right) f_{k}(q, \delta, s) .
$$

Then $\Phi_{k, a}(q, s)$ is independent of $a$ and we may write it as $\Phi_{k}(q, s)$. Furthermore, $\Phi_{k}(q, s)$ is multiplicative function and

$$
\frac{d^{r} \Phi_{k}(q, 1)}{d s^{r}} \ll_{k} q^{-1+\varepsilon}
$$

holds for each integer $r=0,1, \ldots, k-1$.

Proof. First, we have

$$
\begin{aligned}
\Phi_{k, a}(q, s) & =\sum_{\delta \mid q} \sum_{\substack{1 \leq h \leq q \\
\operatorname{gcd}(h, q)=\delta}} e\left(-\frac{a h}{q}\right) f_{k}(q, \delta, s)=\sum_{\delta \mid q} f_{k}(q, \delta, s) \sum_{\substack{1 \leq h_{1} \leq q / \delta \\
\operatorname{gcd}\left(h_{1}, q / \delta\right)=1}} e\left(-\frac{a h_{1}}{q / \delta}\right) \\
& =\sum_{\delta \mid q} c_{\delta}(a) f_{k}(q, q / \delta, s)=\sum_{\delta \mid q} \mu(\delta) f_{k}(q, q / \delta, s),
\end{aligned}
$$

where $c_{\delta}(a)$ is the Ramanujan's sum and we use the fact that if $(a, q / \delta)=(a, q)=1$ then $c_{\delta}(a)=\mu(\delta)$. Therefore $F_{k, a}(q, s)$ is independent on $a$. Suppose that the positive integers $q_{1}$ and $q_{2}$ are coprime, then

$$
\begin{aligned}
\Phi_{k}\left(q_{1}, s\right) \Phi_{k}\left(q_{2}, s\right) & =\sum_{\delta_{2} \mid q_{2}} \sum_{\delta_{1} \mid q_{1}} \mu\left(\delta_{1}\right) \mu\left(\delta_{2}\right) f_{k}\left(q_{1}, q_{1} / \delta_{1}, s\right) f_{k}\left(q_{2}, q_{2} / \delta_{2}, s\right) \\
& =\sum_{\left(\delta_{1} \delta_{2}\right)\left(q_{1} q_{2}\right)} \mu\left(\delta_{1} \delta_{2}\right) f_{k}\left(q_{1}, q_{1} / \delta_{1}, s\right) f_{k}\left(q_{2}, q_{2} / \delta_{2}, s\right),
\end{aligned}
$$

hence we just need to show that

$$
f_{k}\left(q_{1}, q_{1} / \delta_{1}, s\right) f_{k}\left(q_{2}, q_{2} / \delta_{2}, s\right)=f_{k}\left(q_{1} q_{2}, q_{1} q_{2} /\left(\delta_{1} \delta_{2}\right), s\right)
$$

whenever $\delta_{1} \mid q_{1}$ and $\delta_{2} \mid q_{2}$. For this we use the definition of $f_{k}(q, q / \delta, s)$, namely

$$
f_{k}(q, q / \delta, s)=\frac{\delta^{s}}{\varphi(\delta) q^{s}}\left(\sum_{d \mid \delta} \frac{\mu(d)}{d^{s}}\right)^{k} \sum_{d_{1} d_{2} \ldots d_{k}=q / \delta} \sum_{\substack{t_{i}\left(\prod_{j=i+1}^{k} d_{j}\right) \\ \operatorname{gcd}\left(t_{i}, \delta\right)=1 \\ i=1,2, \ldots, k}} \frac{\mu\left(t_{1}\right) \ldots \mu\left(t_{k}\right)}{\left(t_{1} \ldots t_{k}\right)^{s}} .
$$

For $\sigma=\mathfrak{R}(s)$ we obtain

$$
f_{k}(q, q / \delta, s) \ll \frac{\delta^{\sigma}}{\varphi(\delta) q^{\sigma}} \prod_{p \mid \delta}\left(1+\frac{1}{p^{\sigma}}\right)^{k} \sum_{d_{1} d_{2} \ldots d_{k}=q / \delta} \prod_{\substack { i=1 \\
\begin{subarray}{c}{p \mid\left(\prod_{j=i+1}^{k} d_{j}\right) \\
\operatorname{gcd}(p, \delta)=1{ i = 1 \\
\begin{subarray} { c } { p | ( \prod _ { j = i + 1 } ^ { k } d _ { j } ) \\
\operatorname { g c d } ( p , \delta ) = 1 } }\end{subarray}}\left(1+\frac{1}{p^{\sigma}}\right) .
$$

Let us assume that $s$ lies on a circle with a centre $s=1$, so we can write $s=1+\rho e(\theta)$ with $\theta \in[0,1)$ and $\rho \in(0,1)$. Then it is easy to see that

$$
f_{k}(q, q / \delta, s) \ll \frac{\delta^{\sigma}}{\varphi(\delta) q^{\sigma}} 2^{k \omega(\delta)} \tau_{k}(q) 2^{k \omega(q)} \ll q^{\varepsilon} \frac{\delta^{\sigma}}{\varphi(\delta) q^{\sigma}} .
$$


Here $\omega(n)$ is the number of distinct prime factors of $n$ and we used the well known fact that $\omega(n) \ll \frac{\log n}{\log \log n}$ as $n \rightarrow \infty$. Thus we have

$$
\Phi_{k}(q, s) \ll q^{\varepsilon} \sum_{\delta \mid q}|\mu(\delta)| \frac{\delta^{\sigma}}{\varphi(\delta) q^{\sigma}}=q^{-\sigma+\varepsilon} \prod_{p \mid q}\left(1+\frac{p^{\sigma}}{p-1}\right) \ll q^{-\sigma+\varepsilon} \prod_{p \mid q}\left(1+\frac{p^{\sigma}}{p}\right) .
$$

On the other hand, when $\sigma \in(0,2)$, we have

$$
q^{-\sigma} \prod_{p \mid q}\left(1+\frac{p^{\sigma}}{p}\right) \ll \begin{cases}q^{-\sigma+\varepsilon} & \sigma \in(0,1] ; \\ q^{-\sigma+\varepsilon} \prod_{p \mid q} p^{-1+\sigma} \ll q^{-1+\varepsilon} & \sigma \in(1,2) .\end{cases}
$$

Therefore for $\sigma=\mathfrak{R}(s), 0<\sigma<2$, we get

$$
\Phi_{k}(q, s) \ll q^{-\min (\sigma, 1)+\varepsilon} .
$$

It is obvious that $\Phi_{k}(q, s)$ is analytic for every $s \in \mathbb{C}$, and for every parameter $q$ which we consider. Hence one can use Cauchy's integral formula:

$$
\left.\frac{d^{r} \Phi_{k}(q, s)}{d s^{r}}\right|_{s=1}=\frac{r !}{2 \pi i} \int_{|\xi-1|=\rho} \frac{\Phi_{k}(q, \xi)}{(\xi-1)^{r+1}} d \xi \ll \frac{r !}{\rho^{r}} \max _{\theta \in[0,1)}\left|\Phi_{k}(q, 1+\rho e(\theta))\right|,
$$

where $\rho \in(0,1)$. Using (3.2) and choosing $\rho \ll \varepsilon$, we obtain

$$
\frac{d^{r} \Phi_{k}(q, 1)}{d s^{r}} \ll \frac{r !}{\rho^{r}} q^{-(1-\rho)+\varepsilon} \ll_{k, \varepsilon} q^{-1+\varepsilon},
$$

as $q \rightarrow \infty$, which completes the proof of the lemma.

Now we can deal with the representation of the sum $T(\alpha, Y)$.

Proof of Lemma 2.5. First of all, we pick $Y \asymp X^{2}$. Recall that by Lemma 3.1 for $q \leq X^{2 /(k+1)}$ and $\beta=\alpha-a / q$ we have

$$
\begin{aligned}
J_{k}(\alpha, Y) & =\sum_{h=1}^{q} e\left(\frac{a h}{q}\right) \sum_{m=h} \tau_{k \leq X}(m) e(m \beta) \\
& =\sum_{h=1}^{q} e\left(\frac{a h}{q}\right) \int_{0}^{Y} e(u \beta) d\left(M_{k}(u ; h, q)+O_{k}\left(u^{1-\frac{2}{k+1}+\varepsilon}\right)\right) \\
& =\sum_{h=1}^{q} e\left(\frac{a h}{q}\right) \int_{0}^{Y} e(u \beta) M^{\prime}(u ; h, q) d u+O_{k}\left(q(1+|\beta| Y) Y^{1-\frac{2}{k+1}+\varepsilon}\right) .
\end{aligned}
$$

Here we also used a summation formula described for example in [9, Lemma 3.7]. It is clear that

$$
\sum_{h=1}^{q} e\left(\frac{a h}{q}\right) M^{\prime}(u ; h, q)=\sum_{h=1}^{q} e\left(\frac{a h}{q}\right) \operatorname{Res}_{s=1}\left(\zeta(s)^{k} u^{s-1} f_{k}(q, \delta, s)\right),
$$

where $\delta=(q, h)$. This means that

$$
T(\alpha, Y)=\int_{0}^{Y} e(u \beta) \operatorname{Res}_{s=1}\left(\zeta(s)^{k} \Phi_{k}(q, s) u^{s-1}\right) d u+O\left(q(1+|\beta| Y) Y^{1-\frac{2}{k+1}+\varepsilon}\right) .
$$


We now compute $\operatorname{Res}_{s=1}\left(\zeta(s)^{k} \Phi_{k}(q, s) u^{s-1}\right)$. The Riemann zeta function has a Laurent series about $s=1$,

$$
\zeta(s)=\frac{1}{s-1}+\sum_{n=0}^{\infty} \frac{(-1)^{n} \gamma_{n}}{n !}(s-1)^{n},
$$

where

$$
\gamma_{n}=\lim _{M \rightarrow \infty}\left(\sum_{d=1}^{M} \frac{\log ^{n} d}{d}-\frac{\log ^{n+1} M}{n+1}\right), n \in \mathbb{Z}_{\geq 0}
$$

are the Stieltjes constants. Therefore there exist constants

$$
\alpha_{k, j}=\operatorname{Res}_{s=1}\left((s-1)^{j-1} \zeta(s)^{k}\right), j=1,2, \ldots, k,
$$

and a holomorphic function $h_{k}(s)$ on $\mathbb{C}$ such that

$$
\zeta(s)^{k}=\sum_{r=1}^{k} \frac{\alpha_{k, r}}{(s-1)^{r}}+h_{k}(s) .
$$

Thus we obtain that

$$
\zeta(s)^{k} u^{s-1}=\sum_{r=1}^{k} \frac{1}{(s-1)^{r}} \sum_{r_{1}=0}^{k-r} \alpha_{k, r_{1}+r} \frac{\log ^{r_{1}} u}{r_{1} !}+g_{k, u}(s),
$$

for any $u>0$, where $g_{k, u}(s)$ is a holomorphic function on $\mathbb{C}$ about $s$. The Taylor series for $\Phi_{k}(q, s)$ at $s=1$ is

$$
\Phi_{k}(q, s)=\sum_{d=0}^{\infty} \frac{\Phi_{k}^{\langle d\rangle}(q, 1)}{d !}(s-1)^{d}
$$

Therefore the residue of $\zeta(s)^{k} x^{s-1} \Phi_{k}(q, s)$ at $s=1$ is

$$
\sum_{\substack{r-d=1 \\ d, r \in \mathbb{Z}_{+}, 1 \leq r \leq k}} \frac{\Phi_{k}^{\langle d\rangle}(q, 1)}{d !} \sum_{r_{1}=0}^{k-r} \alpha_{k, r_{1}+r} \frac{\log ^{r_{1}} x}{r_{1} !}=\sum_{r=1}^{k} \frac{\log ^{r-1} x}{(r-1) !} \sum_{t=0}^{k-r} \Phi_{k}^{\langle t\rangle}(q, 1) \frac{\alpha_{k, r+t}}{t !} .
$$

Thus if we define

$$
\beta_{k, r}(q)=\frac{1}{r !} \sum_{t=0}^{k-r-1} \frac{1}{t !} \operatorname{Res}_{s=1}\left((s-1)^{r+t} \zeta(s)^{k}\right)\left(\left.\frac{d^{t} \Phi_{k}(q, s)}{d s^{t}}\right|_{s=1}\right)
$$

by Lemma 3.2 we obtain $\beta_{k, r}(q) \ll q^{-1+\varepsilon}$. Furthermore, the error term in (3.3) is

$$
q(1+|\beta| Y) Y^{1-\frac{2}{k+1}+\varepsilon} \ll q(1+L / q) X^{2-\frac{4}{k+1}+\varepsilon} \ll L X^{2-\frac{4}{k+1}+\varepsilon}
$$

for $q \ll L=o\left(X^{\min \left(1, \frac{4}{k+1}\right)}\right)$, which completes the proof of Lemma 2.5. 


\section{THE SINGULAR INTEGRAL AND SERIES}

\subsection{The SINGULAR INTEgRAL}

In this subsection we deal with the singular integral and give a proof of Lemma 2.6. We first proof the following lemmas.

Lemma 4.1. Let $\beta \in \mathbb{R} \backslash\{0\}$ and $Y \geq 2$. We have

$$
\int_{0}^{\gamma} e(-u \beta)(\log u)^{r} d u \ll|\beta|^{-1+\varepsilon} Y^{\varepsilon} .
$$

Proof. We have

$$
\begin{aligned}
\int_{0}^{Y} e(-u \beta)(\log u)^{r} d u & \ll|\beta|^{-1} \int_{0}^{Y|\beta|} e(-u \beta /|\beta|)(\log (u /|\beta|))^{r} d u \\
& \left.\ll|\beta|^{-1} \sum_{\ell=0}^{r}|\log | \beta\right|^{r-\ell}\left|\int_{0}^{Y|\beta|}(\log u)^{\ell} e\left(-u \frac{\beta}{|\beta|}\right) d u\right| \\
& \ll|\beta|^{-1}\left(Y^{\varepsilon}|\beta|^{\varepsilon}+1+\sum_{\ell=1}^{r} \int_{1}^{Y|\beta|} \frac{|\log u|^{\ell-1}}{u} d u\right) \ll|\beta|^{-1+\varepsilon} Y^{\varepsilon} .
\end{aligned}
$$

This completes the proof.

Lemma 4.2. Let $F(\mathbf{t})$ be defined as in (1.1) and $X \geq 2$. If $\beta \in \mathbb{R}$ and $|\beta| \geq X^{-2}$ then

$$
I_{F, \mathcal{B}}(\beta, X):=\int_{X \mathcal{B}} e(F(\mathbf{t}) \beta) d \mathbf{t} \ll|\beta|^{-n / 2+\varepsilon} .
$$

Proof. First, we notice that from the fact that $Q$ is nonsingular it follows that there exists a transformation, such that

$$
\begin{aligned}
\int_{X \mathcal{B}} e(F(\mathbf{t}) \beta) d \mathbf{t} & =\int_{X \mathcal{B}} e\left(\left(\mathbf{t}^{T} Q \mathbf{t}+\mathbf{L}^{T} \mathbf{t}+N\right) \beta\right) d \mathbf{t} \\
& \ll\left|\int_{X \mathcal{B}} e\left(\left(\mathbf{t}^{T} Q \mathbf{t}+\mathbf{L}^{T} \mathbf{t}\right) \beta\right) d \mathbf{t}\right| \ll\left|\int_{X \mathcal{B}+\mathbf{b}_{\mathbf{F}}} e\left(\mathbf{y}^{T} Q \mathbf{y} \beta\right) d \mathbf{y}\right|
\end{aligned}
$$

for some $\mathbf{b}_{\mathrm{F}} \in \mathbb{R}^{n}$. Here $X \mathcal{B}+\mathbf{b}_{\mathrm{F}}$ is still a box, i.e. a factor of intervals, and we can consider that $\mathcal{B}+\mathbf{b}_{\mathrm{F}} / X$ has a maximal side length smaller than 1. According to [1, Lemma 5.2] of Birch, for a quadratic nonsingular form $G$ and a box $\mathfrak{C}$ with a maximal side length smaller than 1 , we have

$$
I_{G, \mathbb{E}}(\beta, 1) \ll|\beta|^{-n / 2+\varepsilon},
$$

where the dependence in this version is uniform on the side length of the box $\mathbb{C}$. Indeed, we apply [1, Lemma 5.2] with $K=n / 2, R=1, d=2$, after we have noticed that the condition (iii) from [1, Lemma 3.2] is not fulfilled for $k=(K-\varepsilon) \Theta$, thus [1, Lemma 4.3] holds in our case too, therefore Lemma 5.2 of Birch applies for our form $Q$. We point out this, since a direct look of the main theorem of Birch implies $n \geq 5$, which is, however, superfluous for [1, Lemma 5.2]. Therefore we have

$$
\int_{X \mathcal{B}+\mathbf{b}_{\mathbf{F}}} e\left(\mathbf{y}^{T} Q \mathbf{y} \beta\right) d \mathbf{y}=I_{Q, \mathcal{B}+\mathbf{b}_{\mathbf{F}} / X}(\beta, X)=X^{-n} I_{Q, \mathcal{B}+\mathbf{b}_{\mathrm{F}} / X}\left(\beta X^{-2}, 1\right) \ll|\beta|^{-n / 2+\varepsilon} X^{-2 \varepsilon} \ll|\beta|^{-n / 2+\varepsilon}
$$

This completes the proof of the lemma. 
Proof of Lemma 2.6. Using Lemma 4.1 and Lemma 4.2, we obtain that

$$
I_{r, F}(\beta, X):=\int_{X \mathcal{B}} d \mathbf{t} \int_{0}^{C_{F, \mathcal{B}}(X)} e((F(\mathbf{t})-u) \beta)(\log u)^{r} d u \ll_{F}|\beta|^{-1-n / 2+\varepsilon} X^{\varepsilon} .
$$

This implies that

$$
\int_{|\beta| \leq L / q X^{2}} I_{r, F}(\beta, X) d \beta=\int_{\mathbb{R}} I_{r, F}(\beta, X) d \beta+O\left(X^{\varepsilon}\left(L / q X^{2}\right)^{-\frac{n}{2}}\right) .
$$

Moreover,

$$
\begin{aligned}
\int_{\mathbb{R}} I_{r, F}(\beta, X) d \beta & =\int_{\mathbb{R}} d \beta \int_{X \mathcal{B}} d \mathbf{t} \int_{0}^{C_{F, \mathcal{B}}(X)} e((F(\mathbf{t})-u) \beta)(\log u)^{r} d u \\
& =2 \int_{\mathbb{R}_{+}} d \beta \int_{0}^{C_{F, \mathcal{B}}(X)}(\log u)^{r} d u \int_{X \mathcal{B}} \cos [2 \pi(u-F(\mathbf{t})) \beta] d \mathbf{t} \\
& =\frac{1}{\pi} \int_{X \mathcal{B}} d \mathbf{t} \int_{\mathbb{R}_{+}} d \beta \int_{0}^{C_{F, \mathcal{B}}(X)}(\log u)^{r} d\left(\frac{\sin [2 \pi(u-F(\mathbf{t})) \beta]}{\beta}\right) \\
& =\frac{1}{\pi} \int_{X \mathcal{B}} d \mathbf{t} \int_{0}^{C_{F, \mathcal{B}}(X)}(\log u)^{r} d\left(\int_{\mathbb{R}_{+}} \frac{\sin [2 \pi(u-F(\mathbf{t})) \beta]}{\beta} d \beta\right) \\
& =\frac{1}{\pi} \int_{X \mathcal{B}} d \mathbf{t} \int_{0}^{C_{F, \mathcal{B}}(X)}(\log u)^{r} d\left(\frac{\pi}{2} \operatorname{sgn}(u-F(\mathbf{t}))\right),
\end{aligned}
$$

where we have used the fact: $\int_{0}^{\infty} \frac{\sin (\alpha x)}{x} \mathrm{~d} x=\frac{\pi}{2} \operatorname{sgn}(\alpha)$ and

$$
\operatorname{sgn}(\alpha):=\left\{\begin{aligned}
\frac{\alpha}{|\alpha|} & \alpha \neq 0 \\
0 & \alpha=0 .
\end{aligned}\right.
$$

By integration by parts we have

$$
\begin{aligned}
\int_{\mathbb{R}} I_{r, F}(\beta, X) d \beta & =\frac{1}{2} \int_{X \mathcal{B}} d \mathbf{t} \int_{0}^{C_{F, \mathcal{B}}(X)}(\log u)^{r} d(\operatorname{sgn}(u-F(\mathbf{t}))) \\
& =\lim _{\epsilon \rightarrow 0^{+}} \int_{X \mathcal{B}} \frac{d \mathbf{t}}{2} \int_{\substack{\mid u \leq-F(\mathbf{t}) \leq \epsilon \\
0 \leq C_{F, \mathcal{B}}(X)}}(\log u)^{r} d(\operatorname{sgn}(u-F(\mathbf{t}))) \\
& \left.=\left.\lim _{\epsilon \rightarrow 0^{+}} \int_{X \mathcal{B}} \frac{d \mathbf{t}}{2}(\log u)^{r}(\operatorname{sgn}(u-F(\mathbf{t})))\right|_{F(\mathbf{t})-\varepsilon} ^{F(\mathbf{t})+\varepsilon}-\int_{\substack{|u-F(\mathbf{t})| \leq \epsilon \\
0 \leq u \leq C_{F, \mathcal{B}}(X)}} \operatorname{sgn}(u-F(\mathbf{t})) d(\log u)^{r}\right) \\
& =\int_{X \mathcal{B}} \frac{1}{2}\left(2(\log F(\mathbf{t}))^{r} d \mathbf{t}+\lim _{\epsilon \rightarrow 0^{+}} O\left(\epsilon \log ^{r} X\right)\right) d \mathbf{t} \\
& =\int_{X \mathcal{B}}(\log F(\mathbf{t}))^{r} d \mathbf{t} .
\end{aligned}
$$

Using (4.1) we get the proof of Lemma 2.6. 


\subsection{THE SINGULAR SERIES}

In this subsection we deal with the singular series, i.e. with the series $L(s ; k, F)$ defined in (2.6), and their presentation stated in Theorem 1.1.

First of all, note that from Lemma 2.4 it follows that $S_{F}(q) \ll q^{1-n / 2+\varepsilon}$ and Lemma 3.2 gives $\frac{d^{r} \Phi_{k}(q, 1)}{d s^{r}} \ll q^{-1+\varepsilon}$ for any integer $r \in[0, k-1]$. Hence, for any $t=0, \ldots, k-1$,

$$
\left.\frac{d^{t} L(s ; k, F)}{d s^{t}}\right|_{s=1}=\sum_{q=1}^{\infty} \frac{d^{t} \Phi_{k}(q, 1)}{d s^{t}} S_{F}(q) \ll \sum_{q=1}^{\infty} q^{-n / 2+\varepsilon} \ll 1,
$$

as $n \geq 3$. By their definition in Theorem 1.1 this ensures that $C_{k, r}(F), r=0, \ldots, k-1$, are convergent and indeed well-defined constants.

It is easily seen that $S_{F}(q)$ defined in (2.5) is real and multiplicative. On the other hand, Lemma 3.2 showed that $\Phi_{k}(q, s)$ is also multiplicative. Therefore $L(s ; k, F)=$ $\sum_{q=1}^{\infty} \Phi_{k}(q, s) S_{F}(q)$ has an Euler product representation as follows:

$$
L(s ; k, F)=\prod_{p} L_{p}(s ; k, F)
$$

with

$$
L_{p}(s ; k, F)=1+\sum_{m \geq 1} S_{F}\left(p^{m}\right) \Phi_{k}\left(p^{m}, s\right)
$$

By orthogonality of characters in $\mathbb{Z} / p^{m} \mathbb{Z}$ for integer $m \geq 1$ it easily follows that

$$
\varrho_{F}\left(p^{m}\right)=p^{-n m} \sum_{1 \leq a \leq p^{m}} S_{F}\left(p^{m}, a\right)
$$

Then we have

$$
S_{F}\left(p^{m}\right)=\varrho_{F}\left(p^{m}\right)-\varrho_{F}\left(p^{m-1}\right)
$$

By the estimate from Lemma 2.4 we get $S_{F}\left(p^{m}\right) \ll_{F}\left(p^{m}\right)^{1-n / 2+\varepsilon}$ and after telescoping summation of (4.2) we obtain

$$
\varrho_{F}\left(p^{\ell}\right)-1 \ll_{F} \sum_{m=1}^{\ell}\left(p^{m}\right)^{1-n / 2+\varepsilon} \ll p^{1-n / 2+\varepsilon},
$$

where we again used that $n \geq 3$. Then by partial summation, using (4.2) and the estimate (3.2), we have

$$
L_{p}(s ; k, F)=\sum_{\ell \geq 0} \varrho_{F}\left(p^{\ell}\right)\left(\Phi_{k}\left(p^{\ell}, s\right)-\Phi_{k}\left(p^{\ell+1}, s\right)\right),
$$

where we set $\varrho_{F}(1)=\Phi_{k}(1, s)=1$.

From (3.1) and the definition of $f_{k}(q, \delta, s)$ in Lemma 3.1, we see that

$$
\begin{aligned}
\Phi_{k}\left(p^{m}, s\right) & =f_{k}\left(p^{m}, p^{m}, s\right)-f_{k}\left(p^{m}, p^{m-1}, s\right) \\
& =\frac{1}{p^{m s}} \sum_{d_{1} d_{2} \ldots d_{k}=p^{m}} \sum_{\substack{t_{i}\left(\prod_{j=i+1}^{k} d_{j}\right) \\
i=1,2, \ldots, k}} \frac{\mu\left(t_{1}\right) \ldots \mu\left(t_{k}\right)}{\left(t_{1} \ldots t_{k}\right)^{s}}-\frac{\left(1-p^{-s}\right)^{k}}{\varphi(p) p^{(m-1) s}} \tau_{k}\left(p^{m-1}\right)
\end{aligned}
$$


For the first expression above, denote

$$
I_{k}=\sum_{d_{1} d_{2} \ldots d_{k}=p^{m}} \sum_{\substack{t_{i}\left(\prod_{j=i+1}^{k} d_{j}\right) \\ i=1,2, \ldots, k}} \frac{\mu\left(t_{1}\right) \ldots \mu\left(t_{k}\right)}{\left(t_{1} \ldots t_{k}\right)^{s}}
$$

Then for $m \geq 1$ and $k=2$ we have

$$
I_{2}=1+m\left(1-p^{-s}\right)
$$

Now using the identities $\tau_{k}\left(p^{m}\right)=\sum_{v=0}^{m} \tau_{k-1}\left(p^{m-v}\right)$, from which it also follows that

$$
\tau_{k}\left(p^{m}\right)-\tau_{k-1}\left(p^{m}\right)=\tau_{k}\left(p^{m-1}\right),
$$

we see that

$$
\begin{aligned}
& I_{k}=\sum_{v=0}^{m} \sum_{d_{1} d_{2} \ldots d_{k-1}=p^{m-v}} \sum_{t_{i}\left(\prod_{j=i+1}^{k} d_{j}\right)} \frac{\mu\left(t_{1}\right) \ldots \mu\left(t_{k}\right)}{\left(t_{1} \ldots t_{k}\right)^{s}}
\end{aligned}
$$

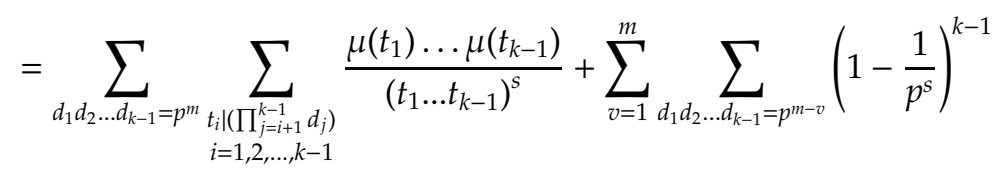

$$
\begin{aligned}
& =I_{k-1}+\left(1-p^{-s}\right)^{k-1} \sum_{v=1}^{m} \tau_{k-1}\left(p^{m-v}\right)=I_{k-1}+\left(1-p^{-s}\right)^{k-1}\left(\tau_{k}\left(p^{m}\right)-\tau_{k-1}\left(p^{m}\right)\right) \\
& =1+m\left(1-p^{-s}\right)+\sum_{v=3}^{k}\left(1-p^{-s}\right)^{v-1} \tau_{v}\left(p^{m-1}\right)=\sum_{v=1}^{k}\left(1-p^{-s}\right)^{v-1} \tau_{v}\left(p^{m-1}\right) .
\end{aligned}
$$

Hence

$$
\Phi_{k}\left(p^{m}, s\right)=p^{-m s}\left(\sum_{1 \leq v \leq k}\left(1-p^{-s}\right)^{v-1} \tau_{v}\left(p^{m-1}\right)-\tau_{k}\left(p^{m-1}\right) \frac{p^{s}\left(1-p^{-s}\right)^{k}}{p-1}\right) .
$$

We now aim to find the value of $\Phi_{k}\left(p^{m}, s\right)-\Phi_{k}\left(p^{m+1}, s\right)$ for each non-negative integer $m$. When $m=1$ we have

$$
\begin{aligned}
\Phi_{k}(1, s)-\Phi_{k}(p, s) & =1-p^{-s}\left(\sum_{v=1}^{k}\left(1-p^{-s}\right)^{v-1} \tau_{v}(1)-\tau_{k}(1) \frac{p^{s}\left(1-p^{-s}\right)^{k}}{p-1}\right) \\
& =1-p^{-s}\left(\frac{1-\left(1-p^{-s}\right)^{k}}{1-\left(1-p^{-s}\right)}-\frac{p^{s}\left(1-p^{-s}\right)^{k}}{p-1}\right) \\
& =\left(1-p^{-s}\right)^{k} \frac{p}{p-1}=\left(1-p^{-1}\right)^{-1}\left(1-p^{-s}\right)^{k} .
\end{aligned}
$$

If $f(z)$ is a formal power series, we denote by $\left[z^{n}\right] f(z)$ the coefficient of $z^{n}$ in $f(z)$. Then for any $|z|<1$ and $m, v \in \mathbb{Z}_{+}$we have

$$
\tau_{v}\left(p^{m-1}\right)=\left[z^{m-1}\right]\left((1-z)^{-v}\right) .
$$


DiVISOR FUNCTION OVER VALUES OF QUADRATIC POLYNOMIALS

Since the symbol $\left[z^{n}\right] f(z)$ has a distributive property, we have

$$
\begin{aligned}
\phi_{k}\left(p^{m}, s\right) & :=\frac{1}{p^{m s}} \sum_{v=1}^{k}\left(1-p^{-s}\right)^{v-1} \tau_{v}\left(p^{m-1}\right) \\
& =\left[z^{m-1}\right]\left(\frac{1}{p^{m s}} \sum_{v=1}^{k} \frac{\left(1-p^{-s}\right)^{v-1}}{(1-z)^{v}}\right) \\
& =\left[z^{m-1}\right]\left(\frac{1}{p^{(m-1) s}} \frac{1}{1-p^{s} z}\left(1-\frac{\left(1-p^{-s}\right)^{k}}{(1-z)^{k}}\right)\right) \\
& =1-p^{(1-m) s}\left(1-p^{-s}\right)^{k}\left[z^{m-1}\right]\left(\left(1-p^{s} z\right)^{-1}(1-z)^{-k}\right) \\
& =1-\left(1-p^{-s}\right)^{k} \sum_{0 \leq \ell \leq m-1} p^{-s \ell}\left[z^{\ell}\right](1-z)^{-k} \\
& =1-\left(1-p^{-s}\right)^{k}\left(\left(1-p^{-s}\right)^{-k}-\sum_{\ell \geq m} p^{-s \ell}\left[z^{\ell}\right](1-z)^{-k}\right) \\
& =\left(1-p^{-s}\right)^{k} \sum_{\ell \geq m} p^{-s \ell}\left[z^{\ell}\right](1-z)^{-k}=\left(1-p^{-s}\right)^{k} \sum_{\ell \geq m} p^{-s \ell} \tau_{k}\left(p^{\ell}\right) .
\end{aligned}
$$

and then for $m \geq 1$ we get

$$
\phi_{k}\left(p^{m}, s\right)-\phi_{k}\left(p^{m+1}, s\right)=\left(1-p^{-s}\right)^{k} p^{-m s} \tau_{k}\left(p^{m}\right) .
$$

From (4.4) it follows that when $m \geq 1$ we have

$$
\begin{aligned}
\Phi_{k}\left(p^{m}, s\right)-\Phi_{k}\left(p^{m+1}, s\right)= & \left(\phi_{k}\left(p^{m}, s\right)-\frac{\left(1-p^{-s}\right)^{k} p^{s}}{p^{s m}(p-1)} \tau_{k}\left(p^{m-1}\right)\right) \\
& -\left(\phi_{k}\left(p^{m+1}, s\right)-\frac{\left(1-p^{-s}\right)^{k} p^{s}}{p^{s(m+1)}(p-1)} \tau_{k}\left(p^{m}\right)\right) \\
= & \left(1-p^{-s}\right)^{k} p^{-m s}\left(\tau_{k}\left(p^{m}\right)-\frac{p^{s}}{p-1}\left(\tau_{k}\left(p^{m-1}\right)-\frac{\tau_{k}\left(p^{m}\right)}{p^{s}}\right)\right) \\
= & \frac{\left(1-p^{-s}\right)^{k}}{1-p^{-1}} p^{-m s}\left(\tau_{k}\left(p^{m}\right)-p^{s-1} \tau_{k}\left(p^{m-1}\right)\right) .
\end{aligned}
$$

Let $\sigma:=\mathfrak{R}(s)>0$. Then according to (3.2) we have $\Phi_{k}\left(p^{\ell}, s\right) \rightarrow 0$, as $\ell \rightarrow 0$ and $s$ is fixed. Then after appropriate telescoping summation we can write

$$
\begin{aligned}
L_{p}(s ; k, F) & =1+\sum_{\ell \geq 0}\left(\varrho_{F}\left(p^{\ell}\right)-1\right)\left(\Phi_{k}\left(p^{\ell}, s\right)-\Phi_{k}\left(p^{\ell+1}, s\right)\right) \\
& =1+\sum_{\ell \geq 1} O\left(p^{1-n / 2+\varepsilon} p^{-\ell \sigma}\left(\tau_{k}\left(p^{\ell}\right)+p^{\sigma-1} \tau_{k}\left(p^{\ell-1}\right)\right)\right)
\end{aligned}
$$

Let us further assume that $\sigma>1 / 2$, so that we obtain

$$
L_{p}(s ; k, F) \ll 1+O\left(p^{1-n / 2+\varepsilon-\sigma}\left(1+p^{\sigma-1}\right)\right)=1+O\left(p^{-n / 2+\varepsilon}+p^{1-n / 2-\sigma+\varepsilon}\right) .
$$

Therefore if $\sigma>\max (1 / 2,2-n / 2)=1 / 2$, and setting $\tau_{k}\left(p^{-1}\right):=0$, we have that the Euler product

$$
L(s ; k, F)=\prod_{p}\left(\sum_{\ell \geq 0} \frac{\varrho_{F}\left(p^{\ell}\right)\left(\tau_{k}\left(p^{\ell}\right)-p^{s-1} \tau_{k}\left(p^{\ell-1}\right)\right)}{p^{\ell s}}\right)\left(\frac{\left(1-p^{-s}\right)^{k}}{1-p^{-1}}\right),
$$


is absolutely convergent. In particular, by (4.3) we have

$$
L(1 ; k, F)=\prod_{p}\left(\sum_{\ell \geq 0} \frac{\varrho_{F}\left(p^{\ell}\right) \tau_{k-1}\left(p^{\ell}\right)}{p^{\ell}}\right)\left(1-\frac{1}{p}\right)^{k-1}>0 .
$$

Now from

$$
C_{k, k-1}=\frac{1}{(k-1) !} L(1 ; k, F) \operatorname{Res}_{s=1}\left((s-1)^{k-1} \zeta(s)^{k}\right)=\frac{L(1 ; k, F)}{(k-1) !}
$$

we conclude that $C_{k, k-1}>0$, which finalizes the proof of Theorem 1.1.

\section{Final Remarks}

We believe that the application of the circle method in estimating divisor sums over values of quadratic polynomials can be extended also to the sum

$$
\Sigma_{k, F}^{\ell}(X ; \mathcal{B}):=\sum_{\mathbf{x} \in X \mathcal{B} \cap \mathbb{Z}^{d}} \tau_{k}^{\ell}(F(\mathbf{x})) .
$$

The treatment of the sum $S(\alpha)$ remains the same, and one could use a level of distribution result for the function $\tau_{k}^{\ell}(m)$ given by Rieger (Satz 3, [14]). In this case a separate treatment for $q$ in the middle range $(\log x)^{\lambda} \leq q \leq L \ll X$ might also be required.

\section{REFERENCES}

[1] B. J. Birch. Forms in many variables. Proc. Roy. Soc. Ser. A, 265:245-263, 1961/1962.

[2] V. Blomer. Higher order divisor problems. Math. Z., 290(3-4):937-952, 2018.

[3] R. de la Bretèche and T. D. Browning. Le problème des diviseurs pour des formes binaires de degré 4 . J. Reine Angew. Math., 646:1-44, 2010.

[4] T. Browning. The divisor problem for binary cubic forms. J. Théor. Nombres Bordeaux, 23(3):579-602, 2011.

[5] S. Daniel. Teilerprobleme für homogene Polynome. Dissertation, Stuttgart, 1997.

[6] S. Daniel. On the divisor-sum problem for binary forms. J. Reine Angew. Math., 507:107-129, 1999.

[7] J. B. Friedlander and H. Iwaniec. A polynomial divisor problem. J. Reine Angew. Math., 601:109-137, 2006.

[8] G. Greaves. On the divisor-sum problem for binary cubic forms. Acta Arith., 17:1-28, 1970.

[9] R. Guo and W. Zhai. Some problems about the ternary quadratic form $m_{1}^{2}+m_{2}^{2}+m_{3}^{2}$. Acta Arith., 156(2):101-121, 2012

[10] L. Hu and L. Yang. Sums of the triple divisor function over values of a quaternary quadratic form. Acta Arith., 183(1):63-85, 2018

[11] H. Liu A divisor problem attached to regular quadratic form. Lith Math J, 59(2):169-184, 2019

[12] Kohji Matsumoto. A remark on Smith's result on a divisor problem in arithmetic progressions. Nagoya Math. J., 98:37-42, 1985.

[13] P. A. B. Pleasants. The representation of primes by quadratic and cubic polynomials. Acta Arith., 12:131$163,1966 / 1967$.

[14] G. J. Rieger. Zum Teilerproblem von Atle Selberg. Math. Nachr., 30:181-192, 1965 


\section{DiVISOR FUNCTION OVER VALUES OF QUADRATIC POLYNOMIALS}

[15] R.A. Smith. The generalized divisor problem over arithmetic progressions. Math. Ann., 260(2):255-268, 1982.

[16] Q. Sun and D. Zhang. Sums of the triple divisor function over values of a ternary quadratic form. J. Number Theory, 168:215-246, 2016.

[17] G. Yu. On the number of divisors of the quadratic form $m^{2}+n^{2}$. Canad. Math. Bull., 43(2):239-256, 2000.

Institute of ANALYsis ANd Number Theory

Graz University of Technology

Kopernikusgasse 24/II

8010 Graz

Austria

lapkova@math.tugraz.at

School of Mathematical Sciences

East China Normal University

500 Dongchuan Road

SHANGHAi 200241

PR CHINA

nianhongzhou@outlook.com 